Progress in Flight Physics 5 (2013) 109-124

DOI: $10.1051 /$ eucass/201305109

(C) Owned by the authors, published by EDP Sciences, 2013

\title{
ARBITRARY LAGRANGIAN-EULERIAN APPROACH IN REDUCED ORDER MODELING OF A FLOW WITH A MOVING BOUNDARY
}

\author{
W. Stankiewicz, R. Roszak, and M. Morzyński \\ Poznan University of Technology \\ Institute of Combustion Engines and Transport \\ 3 Piotrowo, Poznań 60-965, Poland
}

\begin{abstract}
Flow-induced deflections of aircraft structures result in oscillations that might turn into such a dangerous phenomena like flutter or buffeting. In this paper the design of an aeroelastic system consisting of Reduced Order Model (ROM) of the flow with a moving boundary is presented. The model is based on Galerkin projection of governing equation onto space spanned by modes obtained from high-fidelity computations. The motion of the boundary and mesh is defined in Arbitrary LagrangianEulerian (ALE) approach and results in additional convective term in Galerkin system. The developed system is demonstrated on the example of a flow around an oscillating wing.
\end{abstract}

\section{INTRODUCTION}

Interactions between a fluid and moving (deforming) boundaries are among the most important issues of fluid dynamics. They arise in wind turbines [1], civil engineering (e.g., the influence of a wind on bridges and buildings [2, 3]), and the aerospace industry.

In the last case, Fluid-Structure Interaction (FSI) plays an important role in the design process of an aircraft. The examples include dangerous phenomena like flutter and buffeting of the wings and fuselage [4-6], vibrations in turbine engines [7] and helicopter blades [8], as well as applications in the design of bio-inspired air vehicles [9, 10]. Furthermore, recent research on the growth of the lift force and drag reduction by active electromorphing [11] and aeroelastic boundary actuators $[12,13]$ requires the analysis of FSI.

The design of new aircraft requires an analysis of a huge number of variants. One has to check different aircraft configurations, mass cases, gusts, and ma- 
neuvers, giving (even with engineering experience for current configurations and technologies) hundreds of thousands of simulations [14].

One time step of Reynolds-averaged Navier-Stokes (RANS) calculation on the viscous grid using 32-core cluster may take up to $400 \mathrm{~s}$, giving several weeks per simulation. On the other hand, in the case of feedback flow control design the model should be small enough to accurately predict the flow response and ensure that the actuators will work in the correct phase. Long time required to find the flow solution prevents the development of a real-time flow control. This means that further growth in the aerospace industry, leading to more economical and environment-friendly solutions, is possible only through a significant reduction of computation time and memory requirements. Reduced order Galerkin models $[15,16]$ meet these requirements, approximating the governing equations (e.g., Navier-Stokes) with a system of a few ordinary differential equations.

The present paper is organized as follows. The high-dimensional algorithm of FSI is described in section 2. Then, the details on governing equations in ALE approach describing a flow with a moving boundaries (section 3) and the flow model reduction techniques based on Galerkin projection (section 4) are given. In particular, Galerkin Method (subsection 4.1), projection of convective term in ALE approach (subsection 4.2) and Proper Orthogonal Decomposition (POD) used in mode expansion (subsection 4.3) are described. Some remarks on the improvement of model's accuracy are given in section 5. Finally, the ROM of a flow around an oscillating airfoil are presented in section 6 . The results are summarized in section 7 .

\section{FLUID-STRUCTURE INTERACTION ALGORITHM}

Computational Aeroelasticity [13] is a branch of mechanics which examines the interactions between a stream of fluid and a deformable body using the methods of Computational Fluid Dynamics (CFD) and Computational Structural Mechanics (CSM) [6].

The high-dimensional approach used in this work relies on the use of independent solvers for solid and fluid mechanics, exchanging information on the coupling interface. As a result, different discretizations (Finite Volume (FVM) and Finite Element (FEM) Methods) and element types (tetrahedra, plates, beams) are used, and the meshes used on both sides may vary in the number of nodes and elements on the coupling, "wet" surface (interface). Nonconforming grids are the reason of using additional interpolation tools.

The computational FSI algorithm used in this work is shown in Fig. 1.

The velocity and pressure field is calculated by CFD code. In the case of 2D laminar flow, an in-house Direct Numerical Simulation (DNS) solver is used, 
while three-dimensional (3D) Euler/RANS simulations with large numbers of degrees of freedom are performed on parallel and efficient DLR TAU-code [17].

The pressures are interpolated onto the structure using modules based on finite-element meshes, as well as on bucket [18] and oct-tree [19] neighbour search algorithms. Under applied aerodynamic load CSM (elastic) solver calculates the deformations of the structure. The CSM solvers used in this task include an in-house CSM system [20] and open-source Calculix solver [21].

The nodal displacements on the boundary of the structure are

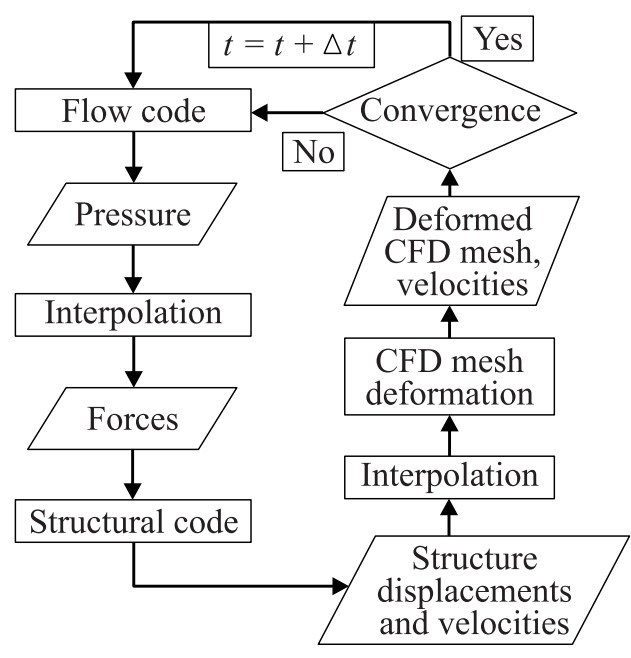

Figure 1 General FSI algorithm interpolated onto CFD mesh. Then the displacements and velocities in the interior of CFD mesh are calculated using a deformation tool based on spring analogy [22].

The flow in modified (deformed) domain results in modified velocity and pressure fields, and different values of structure's node displacements. The loop presented above runs until the convergence in a given time step is reached. Then, the time step is increased using coupling procedures described in [23].

The most time-consuming part of this coupled analysis is a high-fidelity flow solver. To accelerate FSI analyses, e.g., in the aircraft certification procedure and real-time flow control applications, a full-dimensional CFD solver might be replaced by ROM of a flow with moving boundaries.

\section{GOVERNING EQUATIONS}

The viscous, incompressible flow is described by Navier-Stokes equations, that might be written in the form:

$$
\dot{\mathbf{u}}+\nabla \cdot(\mathbf{u} \otimes \mathbf{u})+\nabla \mathbf{p}-\frac{1}{\operatorname{Re}} \Delta \mathbf{u}=0 .
$$

The velocities are defined in respect to the fixed computational mesh. This Eulerian approach widely used in fluid dynamics means that the flow particles move through the mesh elements. This description allows large distortions in 
the fluid motion (separations, vortices, reverse flow, etc.), but it requires precise definition of the domain's boundary.

A technique that overcomes the shortcomings of Eulerian algorithms and allows the simulation of a flow with a moving boundaries is ALE approach [2428]. It combines the best features of Eulerian and Lagrangian approaches by letting the nodes of a fluid mesh move independently of the fluid particles.

In ALE formulation, the velocity of the boundary and the fluid mesh $\mathbf{u}_{\text {grid }}$ is included in the modified convective term:

$$
\dot{\mathbf{u}}+\nabla \cdot\left(\left(\mathbf{u}-\mathbf{u}_{\text {grid }}\right) \otimes \mathbf{u}\right)+\nabla \mathbf{p}-\frac{1}{\operatorname{Re}} \Delta \mathbf{u}=0
$$

where $\mathbf{c}=\mathbf{u}-\mathbf{u}_{\text {grid }}$ is a relative velocity between the material and the mesh and is called convective velocity [28]. The mesh acceleration plays no role in the ALE formulation.

The movement of CFD mesh nodes is independent of the fluid particle motion. In particular, it might be associated with the movement of the structural grid boundary nodes (in Lagrangian approach, equal to the material velocity), ensuring that both CFD and structural meshes will not overlap or disconnect.

In the case of viscous fluid model, the velocity (of fluid particles) on the boundary of the domain is equal to the velocity of the structure (grid) $\left(\mathbf{u}=\mathbf{u}_{\text {grid }}\right)$. In the case of inviscid flows, only the normal components of the velocity are coupled $\left(\mathbf{n} \cdot \mathbf{u}=\mathbf{n} \cdot \mathbf{u}_{\text {grid }}\right)$.

\section{MODEL REDUCTION}

\subsection{Galerkin Method}

In this paper, Galerkin method $[16,29]$ is used to develop ROM that preserves the main flow dynamics. This approach consists in approximation of the velocity field by the base solution $\mathbf{u}_{0}$ (steady or time-averaged flow) and a weighted sum of modes $\mathbf{u}_{j}$ :

$$
\mathbf{u}^{[N]}=\mathbf{u}_{0}+\sum_{j=1}^{N} a_{j} \mathbf{u}_{j}=\sum_{j=0}^{N} a_{j} \mathbf{u}_{j}, \quad a_{0} \equiv 1,
$$

that results in the separation of space $\left(\mathbf{u}_{j}\right)$ and time (mode amplitudes $a_{j}$ ) variables.

The (orthogonal) projection of the residual of approximated Navier-Stokes equation onto the space spanned by the modes:

$$
\left(\mathbf{u}_{i}, R^{[N]}\right)_{\Omega}=\int_{\Omega} \mathbf{u}_{i} R^{[N]} d \Omega=0
$$

results in a system of ordinary differential equations: 


$$
\dot{a}_{i}=\frac{1}{\operatorname{Re}} \sum_{j=0}^{N} a_{j} l_{i j}+\sum_{j=0}^{N} \sum_{k=0}^{N} a_{j} a_{k} q_{i j k}
$$

where

$$
l_{i j}=\left(\mathbf{u}_{i}, \Delta \mathbf{u}_{j}\right)_{\Omega} ; \quad q_{i j k}=-\left(\mathbf{u}_{i}, \nabla \cdot\left(\mathbf{u}_{j} \otimes \mathbf{u}_{k}\right)\right)_{\Omega} .
$$

In Hilbert space, the inner product of two vectors $\mathbf{a}$ and $\mathbf{b}$ is defined as:

$$
(\mathbf{a}, \mathbf{b})_{\Omega}=\int_{\Omega} \mathbf{a b} d \Omega .
$$

\subsection{Projection of Convective Term in Arbitrary Lagrangian-Eulerian Description}

It is assumed that the velocity and displacements of the fluid mesh $\mathbf{u}_{\text {grid }}$ might be decomposed similarly to the velocity of fluid particles (1):

$$
\mathbf{u}_{\text {grid }}=\sum_{j=1}^{N_{G}} a_{j}^{G} \mathbf{u}_{j}^{G}
$$

where modal mesh deformations $\mathbf{u}_{j}^{G}$ are time-invariant.

The projection of the convective term of Navier-Stokes equation in ALE description leads to an additional term in Galerkin system:

$$
\begin{array}{r}
-\left(\mathbf{u}_{i}, \nabla \cdot\left(\left(\mathbf{u}-\mathbf{u}_{\text {grid }}\right) \otimes \mathbf{u}\right)\right)_{\Omega}=- \\
\left(\mathbf{u}_{i}, \nabla \cdot(\mathbf{u} \otimes \mathbf{u})\right)_{\Omega}+\left(\mathbf{u}_{i}, \nabla \cdot\left(\mathbf{u}_{\text {grid }} \otimes \mathbf{u}\right)\right)_{\Omega} \\
=\sum_{j=0}^{N} \sum_{k=0}^{N} q_{i j k} a_{j} a_{k}-\sum_{j=1}^{N_{G}} \sum_{k=0}^{N} q_{i j k}^{G} a_{j}^{G} a_{k}
\end{array}
$$

where $q_{i j k}^{G}=-\left(\mathbf{u}_{i}, \nabla \cdot\left(\mathbf{u}_{j}^{G} \otimes \mathbf{u}_{k}\right)\right)_{\Omega}$.

As the mesh deformation modes are time-invariant, $q_{i j k}^{G}$ term is only affected by the integration over elements of deforming mesh. The changing element shapes might be taken into account using continuous mode interpolation [29, 30].

\subsection{Mode Basis for Model Reduction}

The mode bases used in the Galerkin approximation might be classified in terms of mathematical, physical and empirical approaches, as discussed in [16]. In the empirical approach, the mode basis "is determined a posteriori using experimental or numerical data previously obtained for a given flow configuration" [31]. The possible bases include centroidal Voronoi tessellations (CVT) [32], Lagrange, Hermite and Taylor bases [31], as well as POD [33, 34]. 
Although investigations in the area of empirical modes resulted in modifications of POD method like Sequential POD [35] and Double POD [36] and, recently, Dynamic Mode Decomposition [37, 38], POD is still one of the most popular modeling approaches in fluid dynamics successfully used for flow control and aeroelastic analyses $[16,39,40]$. The POD modes are optimal in energy representation by construction, so they possibly better describe the NavierStokes attractor (limit-cycle oscillations of periodic flow) than the same number of modes obtained in any different manner [41].

In this method, the $M$ flow vectors (snapshots) of the size $N$ (number of degrees of freedom) are centered using time-averaged solution $\mathbf{u}_{0}$ :

$$
\dot{\mathbf{v}}_{i}=\mathbf{v}_{i}-\mathbf{u}_{0}, \quad i=1, \ldots, M .
$$

Resulting $M$ fluctuation vectors $\dot{\mathbf{v}}_{i}$ form a matrix $\dot{V}$. The POD modes used in the model reduction are the eigenvectors $\mathbf{u}_{i}$ of standard eigenproblem $C \mathbf{u}_{i}=\lambda_{i} I \mathbf{u}_{i}$ of the autocorrelation matrix $C$ of size $N \times N$ :

$$
C=\frac{1}{M} \dot{V}^{\mathrm{T}}
$$

related to eigenvalues $\lambda_{i}$ of the largest magnitude.

While the number of snapshots $M$ is substantially smaller than the number of degrees of freedom $N$, a modification of traditional POD proposed by Sirovich [33] is used. In the Method of Snapshots, the autocorrelation matrix $\dot{C}^{\prime}$ of the size $M \times M$ is introduced:

$$
\dot{C}=\frac{1}{M} \dot{V}^{\mathrm{T}} \dot{V} .
$$

The eigenvalues $\lambda$ and $\hat{\lambda}$ of matrices $C$ and $\dot{C}$ are the same, while the eigenvectors (modes) are connected:

$$
\mathbf{u}_{i}=\frac{V \dot{\mathbf{u}}_{i}}{\left\|V \dot{\mathbf{u}}_{i}\right\|}
$$

\section{MODEL CALIBRATION}

The mode basis calculated using POD of a given data set is truncated, and a limited number of the most energetic modes corresponding to the largest eigenvalues $\lambda$ are used in the construction of ROM's mode basis. The neglect of small scales results in filtering of high frequencies and vanishing of energy transfers between resolved and unresolved scales of a fluid flow [42] that decrease the quality of the model. 
The possible inconsistency of data set and the reduced-order formulation (neglect or inaccurate treatment of pressure and boundary terms, not verified incompressibility of the flow) [43], as well as the structural instability of Galerkin Projection $[16,44,45]$ are other sources of discrepancies between Reduced-Order Galerkin model and the high-fidelity model (like DNS of Navier-Stokes equations or Large Eddy Simulation).

The deterioration of model's quality is particularly noticeable in distorted frequencies, phases and amplitudes of mode coefficients, under- or overprediction of the turbulent kinetic energy level and different dynamical responses.

To correct the behavior and improve the accuracy of Reduced Order Galerkin Model, the coefficients of the Galerkin system of ordinary differential equations (ODE) are adjusted [42].

Such a calibration might be done by addition of artificial, "eddy" viscosities to recover the effects of truncated modes [46-48].

This artificial viscosity might be defined as a single, constant value $\nu_{T}$, or $N$ parameters $\nu_{T, i}$ related to each one of the modes:

$$
l_{i j}^{+}=\frac{\nu_{T}}{\nu} l_{i j} ; \quad l_{i j}^{+}=\frac{\nu_{T, i}}{\nu} l_{i j}, \quad i=1, \ldots, N .
$$

Instead of calibrating "eddy" viscosities, all linear coefficients $l_{i j}^{+}[49]$ or both linear and quadratic coefficients $q_{i j k}^{+}$[42] of Galerkin System might be modified in order to improve the results of the calibration.

The resulting system of equations might be written as follows:

$$
\dot{a}_{i}=\underbrace{\nu \sum_{j=0}^{N}\left(l_{i j}+l_{i j}^{+}\right) a_{j}+\sum_{j=0}^{N} \sum_{k=0}^{N}\left(q_{i j k}+q_{i j k}^{+}\right) a_{j} a_{k}}_{f_{i}(\mathbf{a})} .
$$

The model presented above is a subject of the optimization procedure, where objective function related to the prediction error of the model is minimized.

The choices of mean square error of the mode coefficients or their timederivatives, referred as Floquet and Poincaré calibration, respectively, are prevalent $[50,51]$ :

$$
\begin{aligned}
& \chi_{0}:=\sum_{i=1}^{N} \int_{0}^{T}\left(a_{i}^{\mathrm{ROM}}(t)-a_{i}^{\mathrm{DNS}}(t)\right)^{2} d t=\min \\
& \chi_{1}:=\sum_{i=1}^{N} \int_{0}^{T}\left(\dot{a}_{i}^{\mathrm{ROM}}(t)-f_{i}\left(\mathbf{a}^{\mathrm{DNS}}(t)\right)\right)^{2} d t=\min
\end{aligned}
$$


where $a_{i}^{\mathrm{ROM}}$ and $\dot{a}_{i}^{\mathrm{ROM}}$ represent mode coefficients and their time derivatives for Galerkin Model, $a_{i}^{\text {DNS }}$ are the coefficients calculated from POD of reference simulation data, and $f_{i}\left(\mathbf{a}^{\mathrm{DNS}}(t)\right)$ are the values resulting from the substitution of $a_{i}^{\text {DNS }}$ to the function on the right-hand side of Eq. (3).

Another error definitions might be based on the turbulent kinetic energy:

$$
\chi_{2}:=\int_{0}^{T}\left(\sum_{i=1}^{N}\left(a_{i}^{\mathrm{ROM}}(t)\right)^{2}-\sum_{i=1}^{N}\left(a_{i}^{\mathrm{DNS}}(t)\right)^{2}\right)^{2} d t=\min
$$

or modal energy flow balance:

$$
\chi_{3}:=\sum_{i=1}^{N}\left(P_{i}+C_{i}+D_{i}+T_{i}+F_{i}\right)^{2}=\min
$$

(where $P_{i}, C_{i}, D_{i}, T_{i}$, and $F_{i}$ represent the modal production, convection, dissipation, transfer, and pressure power, respectively), leading to E-flow calibration, proposed by Noack et al. [51].

In this work, the optimization procedure based on Genetic Algorithm [52] is used.

\section{RESULTS OF COMPUTATIONS}

\subsection{Two-Dimensional Flow Around NACA-0012 Airfoil}

Two test cases have been chosen for ROM. In the first case, a two-dimensional (2D), incompressible, viscous flow around an oscillating NACA-0012 airfoil is analysed using the in-house DNS solver. The finite-element mesh consists of 5258 nodes and 2558 second-order, 6-node triangular elements.

Reynolds number, related to the chord length, is $\mathrm{Re}=100$, and the angle of attack is $\alpha=15^{\circ}$. The constant inflow velocity is set on the left, top, and bottom edges of the domain. For these parameters, the flow with fixed boundaries has one stable, steady solution. Prescribed sinusoidal transverse oscillations of the airfoil, with an amplitude equal to the one fourth of the chord length and a period equal to $5 \mathrm{~s}$, perturb the flow making it periodic with a bounded amplitude of (limit cycle) oscillations (Fig. 2).

Only one form (transverse direction) of CFD mesh displacements $\mathbf{x}_{\text {grid }}$ and velocities $\mathbf{u}_{\text {grid }}$ makes the separation of space and time variables trivial:

$$
\mathbf{x}_{\text {grid }}=\mathbf{u}_{1}^{G} a_{1}^{G}=\mathbf{u}_{1}^{G} A \sin (f t) ; \quad \mathbf{u}_{\text {grid }}=\mathbf{u}_{1}^{G} \dot{a}_{1}^{G}=\mathbf{u}_{1}^{G} A f \cos (f t)
$$

where $f$ is the frequency and $A$ is the amplitude of the airfoil oscillation. 


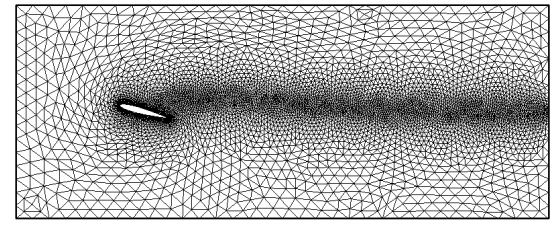

(a)

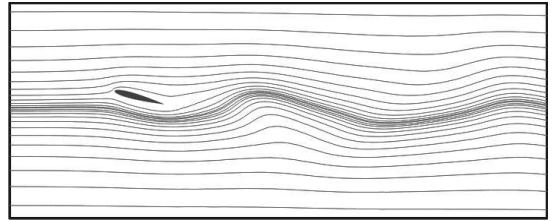

(b)

Figure 2 Finite element mesh for a flow around NACA-0012 airfoil ( $a$ ) and a snapshot of limit-cycle oscillations $(b)$

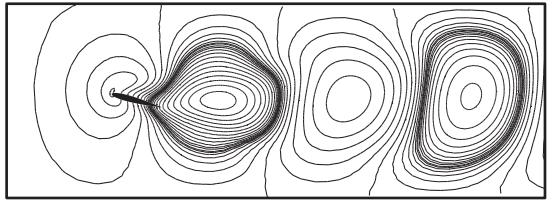

(a)

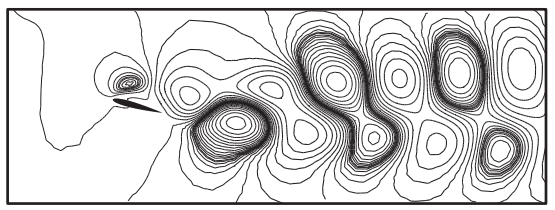

(c)

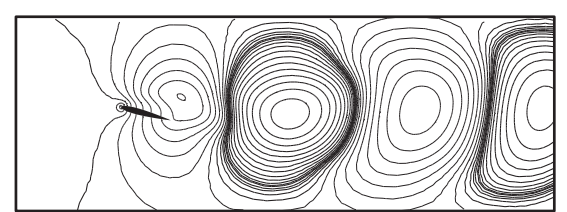

(b)

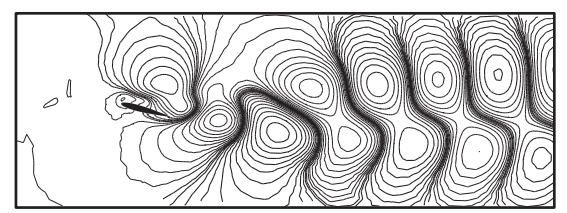

(d)

Figure 3 Streamlines of the most dominant POD modes for a flow around NACA0012 airfoil

The POD has been performed on snapshots from 7 periods of the flow described above. First four of resulting modes, depicted in Fig. 3, carry almost $98 \%$ of information about the kinetic energy of the fluctuation (Table 1).

State equations have been projected onto the space spanned by first eight POD modes (covering $99.97 \%$ of the kinetic energy of the fluctuation). Two Galerkin models have been constructed.

In the first case (Fig. 4a), Galerkin model is formulated in Eulerian approach, neglecting the motion of the boundary and mesh velocities. It can be seen that the initial oscillation is damped, as expected for subcritical values of Reynolds numbers.
Table 1 Information about kinetic energy of fluctuation carried by each POD mode

\begin{tabular}{cc}
\hline Mode & TKE, \% \\
\hline 1 & 51.69 \\
2 & 35.54 \\
3 & 5.59 \\
4 & 5.03 \\
5 & 0.92 \\
6 & 0.90 \\
7 & 0.15 \\
8 & 0.15 \\
\hline
\end{tabular}



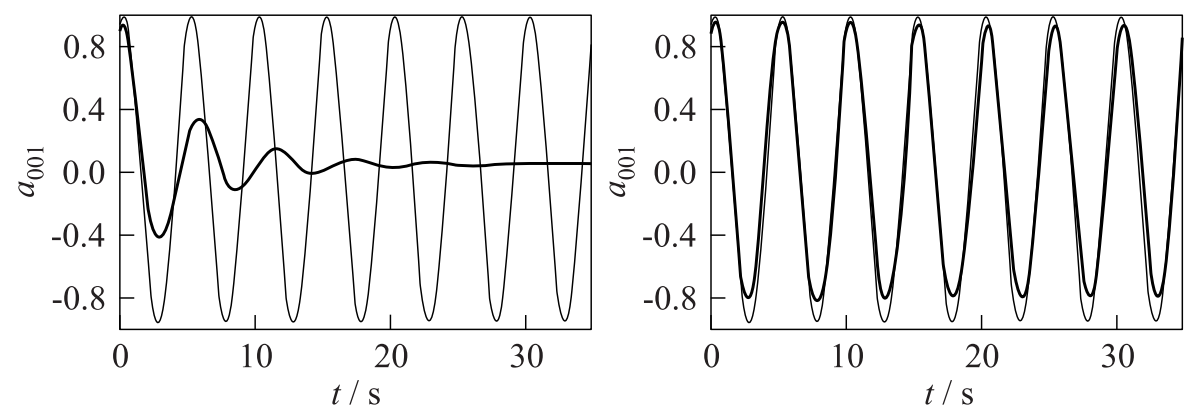

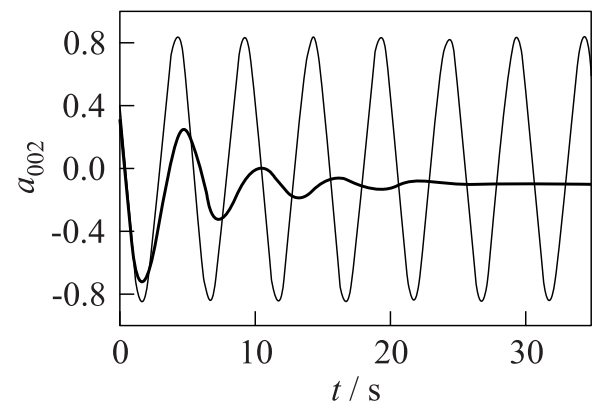

(a)

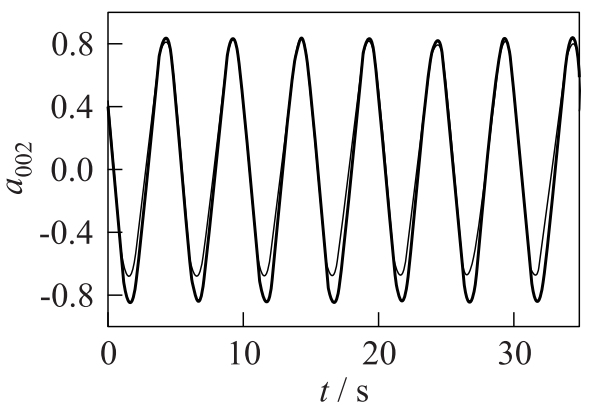

(b)

Figure 4 Amplitudes for first two modes resulting from POD of ALE DNS data (thin lines) and Galerkin models (thick lines). Eulerian ROM neglecting mesh velocities $(a)$ and ALE-based ROM $(b)$ are depicted

It is obvious that both modes basis and approximated governing equations have to be formulated in ALE approach to model the flow with a moving boundary, as is the case of second Galerkin model (Fig. $4 b$ ).

Including the velocity of the mesh in the convective term (2) and proper calibration terms (3), Galerkin model in ALE approach is characterized by the same frequency and almost the same amplitude as reference data from ALEbased DNS.

\subsection{Three-Dimensional Flow Around AGARD 445.6 Wing}

The second test configuration is a generic AGARD 445.6 wing (Fig. 5) analyzed using DLR Tau Code solver based of finite volume method [17].

In this case, the Reynolds number is assumed high enough to neglect boundary layer effects and solve Euler equations. Mach number equals $\mathrm{M}=0.32$ and the angle of attack is $\alpha=0.26^{\circ}$. The reference density is $1.1132 \mathrm{~kg} / \mathrm{m}^{3}$ which corresponds to the altitude of $1 \mathrm{~km}$ above sea level. 


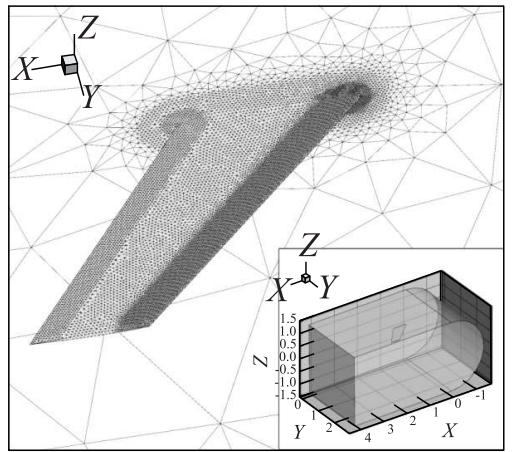

(a)

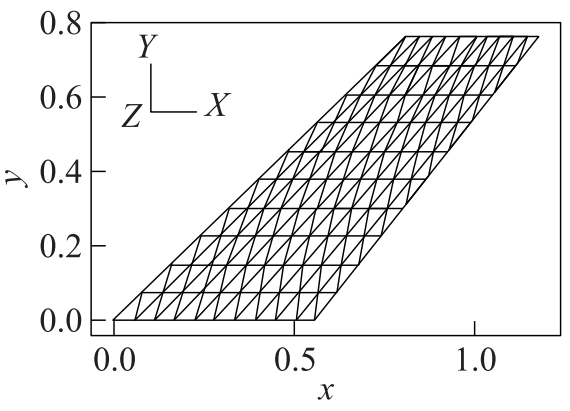

(b)

Figure 5 Computational domain and meshes for CFD $(a)$ and CSM $(b)$ analysis of AGARD 445.6 wing

A D-shaped domain is used for CFD analysis, with symmetry condition on the plane where the wing is mounted and farfield boundary conditions on the opposite one, as well as top and bottom planes.

The CFD mesh consists of 319,919 nodes and 1,873,626 first-order tetrahedral elements. The structure is modeled as a "plate model" consisting of 121 nodes, 200 triangular plate elements with varying thickness, and, additionally, 20 beam elements on the leading and trailing edges.

The deformations of the structure under an aerodynamic load are calculated using the in-house FEM solver. For a given configuration, the growth of the oscillation's amplitude is observed (Fig. 6).

The snapshots from 4 periods of oscillations (see Fig. 6b) computed using a high-fidelity aeroelastic system have been decomposed using POD. The most

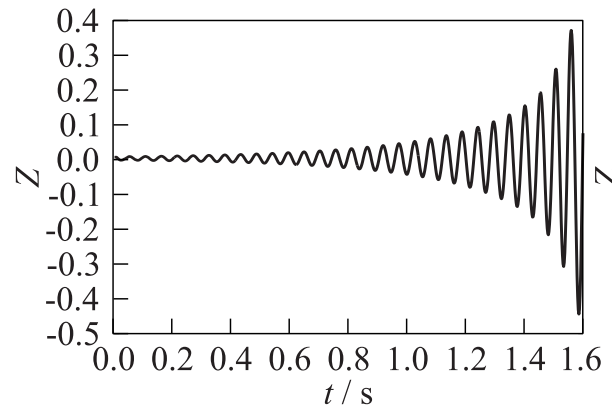

(a)

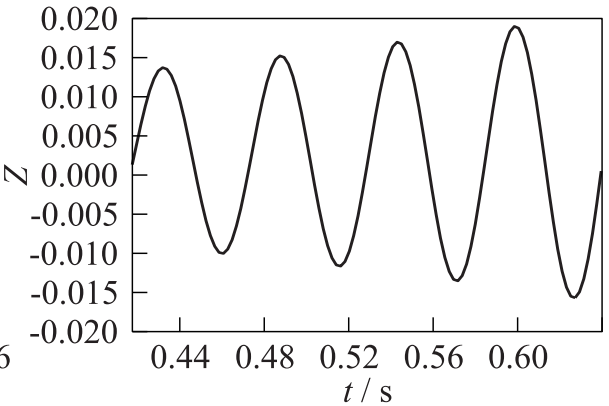

(b)

Figure $6 Z$-component of the displacement of a node on the end of a trailing edge of the AGARD wing 

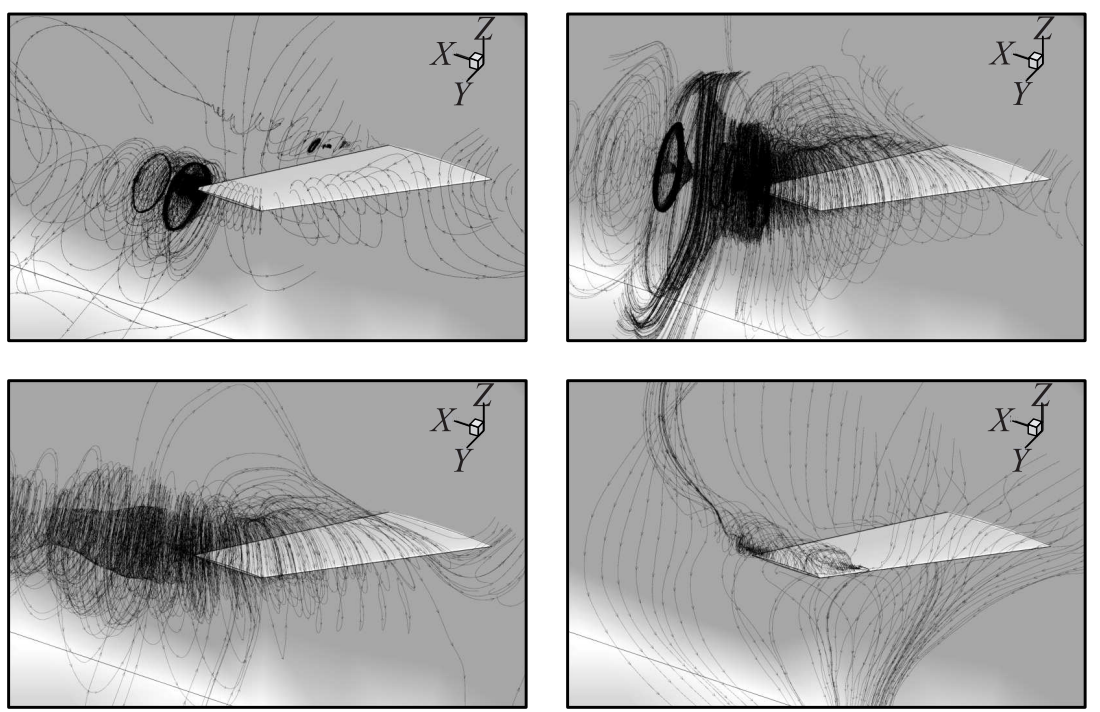

Figure 7 Streamlines of the most dominant POD modes for a flow around AGARD 445.6 wing

Table 2 Information about kinetic energy of fluctuation carried by each POD mode

\begin{tabular}{cc}
\hline Mode & TKE, $\%$ \\
\hline 1 & 98.10 \\
2 & 1.26 \\
3 & 0.12 \\
4 & 0.05 \\
\hline
\end{tabular}

energetic of resulting modes, representing $99.9 \%$ of kinetic energy of fluctuation, are depicted in Fig. 7. It can be seen that for Euler flow with growing amplitude of oscillation, as opposed to DNS of Navier-Stokes equations, POD modes do not form pairs. Additionally, the first POD mode carries $98 \%$ of the fluctuation energy, compared to $1 \%$ of the second mode (Table 2 ).

The projection of the flow snapshots onto the space spanned by POD modes allows to calculate the reference values of mode amplitudes. For the first two modes, they are depicted in Fig. 8.

The resulting functions are characterized by the same frequency and growth rate as the graph of node displacements (see Fig. $6 b$ ).

\section{SUMMARY}

In this paper, high-fidelity aeroelastic simulations and ROMs of a flow with a moving boundary are presented.

Arbitrary Lagrangian-Eulerian approach allows, by introducing convective velocity into the governing equations, the modeling of a rapid boundary move- 


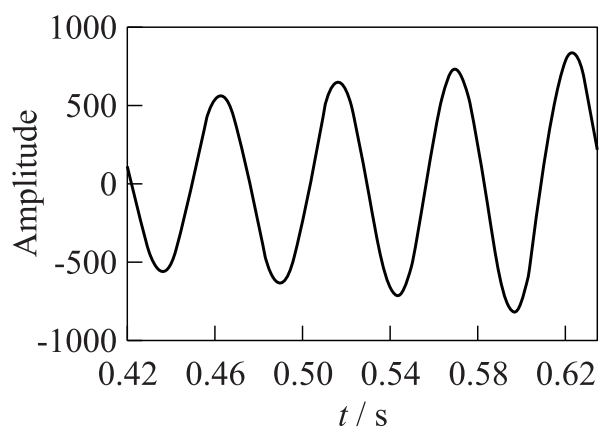

(a)

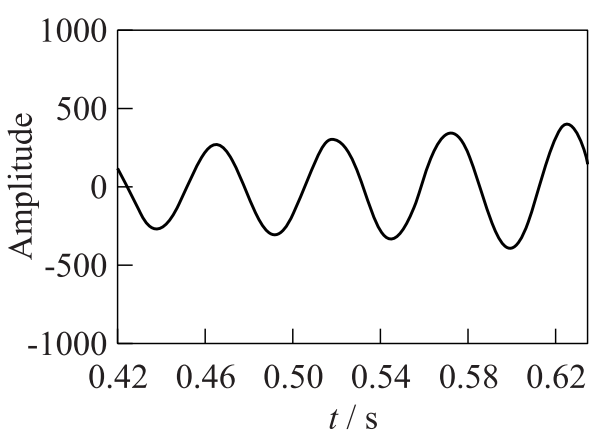

(b)

Figure 8 Amplitudes for first two modes resulting from POD of reference data: (a) mode 1 ; and $(b)$ mode 2

ment similar to the airfoil and wing oscillation. Galerkin projection of governing equations (either Navier-Stokes or Euler) in ALE formulation results in additional quadratic term $q_{i j k}^{G}$ in the Galerkin system representing triadic interactions between two "flow" modes and one "mesh velocity" mode.

In this paper, mode bases resulting from POD of numerical simulation data have been used. The mode basis construction for the reduced order modeling has been demonstrated on a $2 \mathrm{D}$ viscous flow around a NACA-0012 airfoil and a $3 \mathrm{D}$ inviscid flow around a generic AGARD 445.6 wing.

It has been shown that the design of ROMs of the flows with a moving boundary is possible using ALE approach.

Further investigations include the extension of the range of applicability of the model by parametrization of mode bases using Continuous Mode Interpolation [30]. Such approach will allow modeling of the flow under varying operating conditions, like different Mach numbers and flight altitudes.

Additionally, mode basis resulting from Dynamic Mode Decomposition [37, 38 ] will be tested in the cases where the limit cycle oscillation is not reached.

\section{ACKNOWLEDGMENTS}

This work has been funded by the Polish Ministry of Science under research grant No. N N501 225437.

We thank Bernd R. Noack (CNRS, Université de Poitiers) and Gilead Tadmor (Northeastern University, Boston) for very stimulating discussions. 


\section{REFERENCES}

1. Hansen, M. O.L., J. N. Sørensen, S. Voutsinas, N. Sørensen, and H. A. Madsen. 2006. State of the art in wind turbine aerodynamics and aeroelasticity. Prog. Aerospace Sci. 42(4):285-330.

2. Katsuchi, H., N.P. Jones, and R. H. Scanlan 1999. Multimode coupled flutter and buffeting analysis of the Akashi-Kaikyo Bridge. J. Struct. Eng. 125:60.

3. Stathopoulos, T., and C.C. Baniotopoulos. 2007. Wind effects on buildings and design of wind-sensitive structures. Springer Verlag.

4. Farhat, C. 2004. CFD-based nonlinear computational aeroelasticity. In: Encyclopedia of computational mechanics. 3:459-80.

5. Wright, J. R., and J. E. Cooper. 2007. Introduction to aircraft aeroelasticity and loads. Wiley.

6. Roszak, R., P. Posadzy, E. Stankiewicz, M. Morzyński. 2009. Fluid structure interaction for large scale complex geometry and non-linear properties of structure. Archives Mech. 61(1):3-27.

7. Srinivasan, A. V. 1997. Flutter and resonant vibration characteristics of engine blades. J. Eng. Gas Turbines Power 119(4):742-75.

8. Leishman, J. G. 2006. Principles of helicopter aerodynamics. Cambridge: Cambridge University Press.

9. DeLaurier, J. D. 1993. The development of an efficient ornithopter wing. Aeronautical J. 97:153.

10. Carruthers, A. C., A. L. R. Thomas, and G. K. Taylor. 2007. Automatic aeroelastic devices in the wings of a steppe eagle aquila nipalensis. J. Exp. Biol. 210(23):4136.

11. Nogarede, B., C. Henaux, J. F. Rouchon, and E. Duhayon. 2006. Electroactive materials: From piezomotors to electroactive morphing. IEEE Industrial Electronics, IECON 2006 - 32nd Annual Conference. 4437-41.

12. Livne, E. 2003. Future of airplane aeroelasticity. J. Aircraft 40(6):1066-92.

13. Kamakoti, R., and W. Shyy. 2004. Fluid-structure interaction for aeroelastic applications. Prog. Aerospace Sci. 40(8):535-58.

14. Rossow, C., and N. Kroll. 2008. High performance computing serves aerospace engineering: Opportunities for next generation product development. 46th AIAA Aerospace Science Meeting and Exhibit. Reno, USA.

15. Gerhard, J., M. Pastoor, R. King, B. R. Noack, A. Dillmann, M. Morzyński, and G. Tadmor. 2003. Model-based control of vortex shedding using low-dimensional Galerkin models. AIAA Paper No. 2003-4262.

16. Noack, B. R., K. Afanasiev, M. Morzyński, G. Tadmor, and F. Thiele. 2003. A hierarchy of low-dimensional models for the transient and post-transient cylinder wake. J. Fluid Mech. 497:335-63.

17. Schwamborn, D., T. Gerhold, and R. Heinrich. 2006. The DLR TAU-code: Recent applications in research and industry. European Conference on Computational Fluid Dynamics, ECCOMAS CFD.

18. Benson John, O., and J. David. 1990. A single surface contact algorithm for the post-buckling analysis of shell structures. Comput. Meth. Appl. Mech. Eng. 78(2):141-63. 
19. Löhner, R. 1988. Some useful data structures for the generation of unstructured grids. Comm. Appl. Num. Meth. 4(1):123-35.

20. Morzyński, M. 1987. Numerical solution of Navier-Stokes equations by the finite element method. SYMKOM 87 Proceedings, Compressor and Turbine Stage Flow Path - Theory and Experiment. 119-28.

21. Dhondt, G. 2004. The finite element method for three-dimensional thermomechanical applications. John Wiley \& Sons, Ltd.

22. Farhat, C., C. Degand, B. Koobus, and M. Lesoinne. 1998. Torsional springs for two-dimensional dynamic unstructured fluid meshes. Comput. Meth. Appl. Mech. Eng. 163(1-4):231-45.

23. Piperno, S., C. Farhat, and B. Larrouturou. 1995. Partitioned procedures for the transient solution of coupled aroelastic problems. Part I: Model problem, theory and two-dimensional application. Comput. Meth. Appl. Mech. Eng. 124(1-2):79112.

24. Donea, J., S. Giuliani, and J. P. Halleux. 1982. An arbitrary Lagrangian-Eulerian finite element method for transient dynamic fluid-structure interactions. Comput. Meth. Appl. Mech. Eng. 33:689-723.

25. Lin, W. K., H. Chang, J. S. Chen, and T. Belytschko. 1988. Arbitrary Lagrangian Eulerian Petrov-Galerkin finite elements for nonlinear continua. Comput. Meth. Appl. Mech. Eng. 68:259-310.

26. Willcox, K. E. 2000. Reduced-order aerodynamic models for aeroelastic control of turbomachines. Ph.D. Thesis. Massachusetts Institute of Technology.

27. Sarrate, J., A. Huerta, and J. Donea. 2001. Arbitrary Lagrangian-Eulerian formulation for fluid-rigid body interaction. Comput. Meth. Appl. Mech. Eng. 190:317188 .

28. Donea, J., A. Huerta, J.-Ph. Ponthot, and A. Rodríguez-Ferran. 2004. Arbitrary Lagrangian-Eulerian method. In: Encyclopedia of computational mechanics. Vol. 1: Fundamentals. Eds. E. Stein, R. de Borst, and T. J. R. Hughes. John Wiley and Sons, Ltd.

29. Stankiewicz, W., M. Morzyński, R. Roszak, B. R. Noack, and G. Tadmor. 2008. Reduced order modelling of a flow around an airfoil with a changing angle of attack. Archives Mech. 60(6)509-26.

30. Morzyński, M., W. Stankiewicz, B. R. Noack, R. King, F. Thiele, and G. Tadmor. 2007. Continuous mode interpolation for control-oriented models of fluid flow. In: Notes on numerical fluid mechanics and multidisciplinary design. Vol. 95: Active flow control. Ed. R. King. Berlin, Germany: Springer. 260-78.

31. Bergmann, M., L. Cordier, and J.-P. Brancher. 2005. Optimal rotary control of the cylinder wake using proper orthogonal decomposition reduced order model. Phys. Fluids. 17:1-21.

32. Burkardt, J., M. Gunzburger, and H. C. Lee. 2007. Centroidal Voronoi tessellationbased reduced-order modeling of complex systems. SIAM J. Scientific Computing 28(2):459-84.

33. Sirovich, L. 1987. Turbulence and the dynamics of coherent structures. Part I: Coherent structures. Quart. Appl. Math. XLV:561-71.

34. Holmes, P., J. L. Lumley, and G. Berkooz. 1998. Turbulence, coherent structures, dynamical systems and symmetry. Cambridge: Cambridge University Press. 
35. Jørgensen, B. H., J. N. Sørensen, and M. Brøns. 2003. Low-dimensional modeling of driven cavity flow with two free parameters. Theoret. Comp. Fluid Dyn. 16:299317.

36. Siegel, S. G., J. Seidel, C. Fagley, D. M. Luchtenburg, K. Cohen, and T. McLaughlin. 2008. Low-dimensional modelling of a transient cylinder wake using double proper orthogonal decomposition. J. Fluid Mech. 610:1-42.

37. Schmid, P. J., and J. Sesterhenn. 2010. Dynamic mode decomposition of numerical and experimental data. J. Fluid Mech. 656:5-28.

38. Frederich, O., and D. M. Luchtenburg. 2011. Modal analysis of complex turbulent flow. 7th Symposium (International) on Turbulence and Shear Flow Phenomena.

39. Dowell, E. H., J. P. Thomas, and K. C. Hall. 2004. Transonic limit cycle oscillation analysis using reduced order aerodynamic models. J. Fluid Struct. 19:17-27.

40. Lieu, T., C. Farhat, and M. Lesoinne. 2006. Reduced-order fluid/structure modeling of a complete aircraft configuration. Comput. Meth. Appl. Mech. Eng. 195:5730-42.

41. Noack, B. R. 2005. Niederdimensionale Galerkin-Modelle für laminare und transitionelle Scherströmungen transl.: Low-dimensional Galerkin models of laminar and transitional shear-flow). Technical Report. Habilitation thesis.

42. Couplet, M., C. Basdevant, and C. Sagaut. 2005. Calibrated reduced-order PODGalerkin system for fluid flow modelling. J. Comp. Phys. 207(1):192-220.

43. Cordier, L., B. Abou El Majd, and J. Favier. 2009. Calibration of POD reducedorder models using Tikhonov regularization. Int. J. Numer. Meth. Fluids 63(2):26996.

44. Iollo, A., S. Lanteri, and J. A. Désidèri. 1998. Stability properties of POD-Galerkin approximations for the compressible Navier-Stokes equations. Technical Report 3589. INRIA.

45. Rempfer, D. 2000. On low-dimensional Galerkin models for fluid flow. Theor. Comput. Fluid Dyn. 14:75-88.

46. Aubry, N., P. Holmes, J. L. Lumley, and E. Stone. 1988. The dynamics of coherent structures in the wall region of a turbulent boundary layer. J. Fluid Mech. 192:11573.

47. Rempfer, D. 1991. Kohärente Strukturen und Chaos beim laminar-turbulenten Grenzschichtumschlag (transl.: Coherent structures and chaos of the laminarturbulent boundary-layer transition). Fakultät Verfahrenstechnik der Universität Stuttgart. (Part of this work has been published by Rempfer, D., and F. H. Fazle. 1994. J. Fluid Mech. 260-75.)

48. Podvin, B. 2001. On the adequacy of the ten-dimensional model for the wall layer. J. Phys. Fluids 13:210-24.

49. Galletti, G., C. H. Bruneau, L. Zannetti, and A. Iollo. 2004. Low-order modelling of laminar flow regimes past a confined square cylinder. J. Fluid Mech. 503:161-70.

50. Bourguet, R., M. Braza, A. Dervieux, and A. Sévrain. 2007. Transition features in transonic flow around a naca0012 airfoil by Navier-Stokes and low-order modeling. West-East High Speed Flow Field ECCOMAS Conference Proceedings. Moscow, Russia.

51. Noack, B. R., M. Morzyński, and G. Tadmor. 2011. Reduced-order modelling for flow control. In: CISM courses and lectures 528. Springer-Verlag.

52. Stankiewicz, W., R. Roszak, and M. Morzyński. 2011. Genetic algorithm-based calibration of reduced order Galerkin models. Math. Modell. Anal. 16(2):233-47. 